\title{
Dynamic vaccination in partially overlapped multiplex network
}

\author{
L. G. Alvarez-Zuzek* and M. A. Di Muro \\ Departamento de Física, Facultad de Ciencias Exactas y Naturales, Universidad Nacional de Mar del Plata, \\ and Instituto de Investigaciones Físicas de Mar del Plata (IFIMAR-CONICET), Deán Funes 3350, 7600 Mar del Plata, Argentina
}

S. Havlin

Department of Physics, Bar-Ilan University, Ramat-Gan 52900, Israel

L. A. Braunstein

Departamento de Física, Facultad de Ciencias Exactas y Naturales, Universidad Nacional de Mar del Plata, and Instituto de Investigaciones Físicas de Mar del Plata (IFIMAR-CONICET), Deán Funes 3350, 7600 Mar del Plata, Argentina and Center for Polymer Studies, Boston University, Boston, Massachusetts 02215, USA

(Received 27 April 2018; published 2 January 2019)

\begin{abstract}
In this work we propose and investigate a strategy of vaccination which we call "dynamic vaccination." In our model, susceptible people become aware that one or more of their contacts are infected and thereby get vaccinated with probability $\omega$, before having physical contact with any infected patient. Then the nonvaccinated individuals will be infected with probability $\beta$. We apply the strategy to the susceptible-infected-recovered epidemic model in a multiplex network composed by two networks, where a fraction $q$ of the nodes acts in both networks. We map this model of dynamic vaccination into bond percolation model and use the generating functions framework to predict theoretically the behavior of the relevant magnitudes of the system at the steady state. We find a perfect agreement between the solutions of the theoretical equations and the results of stochastic simulations. In addition, we find an interesting phase diagram in the plane $\beta$ - $\omega$, which is composed of an epidemic and a nonepidemic phase, separated by a critical threshold line $\beta_{c}$, which depends on $q$. As $q$ decreases, $\beta_{c}$ increases, i.e., as the overlap decreases, the system is more disconnected, and therefore more virulent diseases are needed to spread epidemics. Surprisingly, we find that, for all values of $q$, a region in the diagram where the vaccination is so efficient that, regardless of the virulence of the disease, it never becomes an epidemic. We compare our strategy with random immunization and find that, using the same amount of vaccines for both scenarios, we obtain that the spread of disease is much lower in the case of dynamic vaccination when compared to random immunization. Furthermore, we also compare our strategy with targeted immunization and we find that, depending on $\omega$, dynamic vaccination will perform significantly better and in some cases will stop the disease before it becomes an epidemic.
\end{abstract}

DOI: 10.1103/PhysRevE.99.012302

\section{INTRODUCTION}

In 2009, the pandemic virus (H1N1) was identified as the cause of many cases of human illnesses in California and Texas and a severe outbreak in Mexico [1-3]. The pandemic had a reproduction value of approximately 1.5 and appeared to exhibit a community transmissibility similar to the respiratory pathogen Severe Acute Respiratory Syndrome (SARS) coronavirus (SARS-CoV) [4-6]. Even though the most commonly affected age group was 5-45 years old, the influenza also affected other age groups, such as older adults, pregnant women, and children. By the end of the pandemic in 2010, it was registered that the virus caused the death of around 20000 people all over the world, and it was fueled by the mobility between regions and different countries. In Ref. [7] the authors studied the role of travel restrictions in halting pandemics by using short-range mobility data and explored

\footnotetext{
*1galvere@mdp.edu.ar
}

alternative scenarios by assessing the potential impact of mobility restrictions. However, although this strategy could be very effective, it was found only useful to slow down the spread of diseases, which might give time to the health authorities to develop a better strategy to stop the epidemic, for example, the development of a new vaccine. Fortunately, in the case of the H1N1 pandemic, it was possible to develop and deploy a vaccination campaign just in time to prevent further spread of disease.

It is well known that infectious diseases usually spread by physical contact between individuals in a society $[8,9]$. Over the years, researchers have found that the best way to model these types of contact patterns [10-12] is by using the topology of complex networks [13-17], where people are represented by nodes and their interactions as links. A commonly used model for reproducing the dynamics of the spreading of endemic diseases, such as seasonal influenza or SARS [6], is the susceptible-infected-recovered (SIR) model [18-20]. This model groups individuals of a population into three compartments according to their state: susceptible (S), 
infected (I), and recovered (R). When a susceptible individual is in contact with an infected one, it becomes infected with probability $\beta$, which is the same for everyone. Infected individuals recover after a period of time $t_{r}$, i.e., they become immunized and cannot be infected again or infect others. When the parameters $\beta$ and $t_{r}$ are constant, the effective probability of infection is given by the transmissibility $T=$ $1-(1-\beta)^{t_{r}}[21,22]$. The SIR model has a treelike structure with branches of infection that develop and expand, and this is because infected individuals cannot be reinfected, so the infection can only move forwards. It has been proven that this process can be mapped into link percolation [23,24], and thus the dynamic can be described using the generating function framework. The most important property in this framework is the probability $f$ that a branch of infection will expand throughout the network [24]. When a branch of infection reaches a node with $k$ connections across one of its links, it can only expand through its $k-1$ remaining connections. It can be shown that $f$ satisfies the transcendental equation $f=1-$ $G_{1}(1-T f)$, where $G_{1}(x)=\sum_{k=k_{\min }}^{k_{\max }} k P(k) /\langle k\rangle x^{k-1}$, for $x \in$ $[0,1]$, is the generating function of the underlying branching process [23]. Note that $G_{1}(1-T f)$ represents the probability that the branches of infection do not expand throughout the network. In the steady state of this process, there is a critical threshold $T_{c}$ that separates an epidemic phase from a nonepidemic phase. When $T \leqslant T_{c}$ there is an epidemicfree phase with only small outbreaks, which corresponds to finite clusters in link percolation theory. But, when $T>T_{c}$ an epidemic phase develops, the branches of infection contribute to a spanning cluster of recovered individuals. Thus, the probability of selecting a random node that belongs to the spanning cluster is given by $R=1-G_{0}(1-T f)$, where $G_{0}(x)=\sum_{k=k_{\min }}^{k_{\max }} P(k) x^{k}$ is the generating function of the degree distribution.

The spread of epidemics in networks [25-28] have been the focus of motivation of several investigations that seek to develop and study different strategies of mitigation for decreasing the impact of diseases on healthy populations [18,29-33]. On one hand, referring to nonpharmaceutical strategies, one of the most common and studied is "quarantine," in which all individuals of the affected population must remain in isolation for a period of time. This scenario is difficult to perform, besides it involves a great economic loss. On the other hand, a more moderate strategy proposed is "social distancing" [34-36]. In this strategy, susceptible individuals distance themselves from infected or from those having the symptoms of the disease by removing links to them. Although all these strategies are beneficial, without any doubt, the most effective one is "vaccination" [37] (and references therein). In the early times random vaccination has been studied [38]. In this protocol susceptible individuals are vaccinated regardless of whether they are in contact with an infected individual, which requires a huge amount of vaccines and resources that may not be available. One way to improve this strategy is targeted vaccination [39], that is, to vaccinate those people who have many connections and therefore higher probability of getting the disease and transmitting it. However, this strategy is difficult to implement, since the degrees of nodes in the network must be known in detail. In Ref. [40], the authors proposed the acquaintance immunization strategy which does not require knowledge of degrees. In the model, a fraction of nodes is chosen at random, then some of their closest contacts get immunized. So, given that in scale-free networks a randomly chosen link points with high probability to a high degree node, this strategy is actually a preferential immunization of the hubs, which reduces dramatically the amount of vaccines needed to control the epidemic.

Since the advent of multilayer networks or network of networks these structures have been the focus of much research and have allowed the scientific community to use a more realistic approach. With this new insight, the spread of epidemics was once again an important subject to investigate [32,41-45]. On top of that, the cases of the H1N1 pandemic (2009) and the Ebola outbreak in Africa (2014), and their catastrophic consequences worldwide, have prompted the research of new mitigation strategies to avoid similar future epidemic outbreaks [36,46-50]. With this motivation in mind, we develop a vaccination strategy which we called "dynamic vaccination" on the topology of a multiplex network. We are interested in studying how epidemic spreads in the presence of this vaccination protocol. In our model, the group of susceptible people that have a relation with infected individuals is the target of the immunization strategy. The contacts or neighbors of an infected person receives a vaccine with a probability $\omega$. If the susceptible individuals are vaccinated, then they acquire immunization and cannot be infected or infect others anymore.

This strategy, known also in the epidemiology field as "ring vaccination," has been applied to eradicate the smallpox [51] and has been studied in Refs. [52,53] due to its efficiency as a vaccination protocol. It has been also implemented during the Ebola virus epidemic in West Africa $(2015)$ [54,55]. In this article we present insights by applying the strategy on the SIR epidemic model with the topology of a multiplex network. We perform stochastic simulations and we apply the generating functions framework to develop theoretical equations that describe the outcome of the model. We find an excellent agreement between the simulation results and the solutions of the analytical equations.

This manuscript is organized as follows: Section II explains the model and the results obtained, and this section is divided in three parts, $A, B$, and $C$. In $A$ we present the rules of the epidemic model with the dynamic vaccination. Then, in $B$ we develop the theory that corresponds to the epidemic spreading problem. Last, in $C$ we show the simulations results and compare them with the results of the theoretical equations, which are solved numerically. Finally, in Sec. III we present our discussions and outlooks.

\section{MODEL AND RESULTS}

Our epidemic model is performed on a multiplex system composed of two networks $A$ and $B$, each one characterized by a degree distribution $P^{\alpha}(k)$, with $\alpha=A, B$. Both networks are connected to each other through a fraction $q$ identical pairs of nodes in both networks [44]. Those pairs of nodes in network $A$ and $B$ represent the same individual acting in different networks. For example, one network could represent the personal contacts of the individuals at their workplace and the other network their family or friends. 

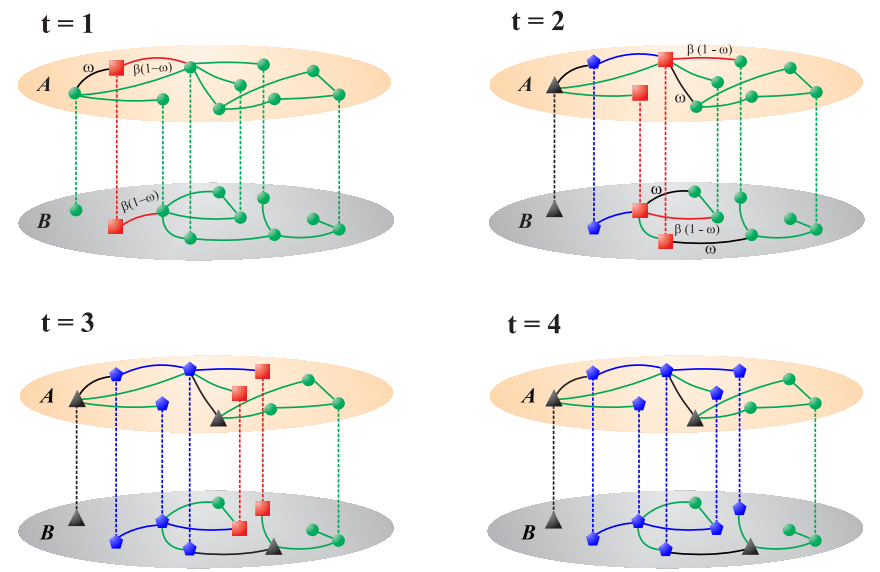

FIG. 1. Schematic of the SI-R/V epidemic process in a multiplex network consisting of two networks, each of size $N=10$. A fraction $q$ of nodes in both networks represent the same individual acting in different environments. Here $q=0.7$. The colors of the nodes represent the following states: green $(\bullet)$ for susceptible $(S)$, red (ם) for infected (I), blue ( $)$ for recovered (R), and black $(\boldsymbol{\Delta})$ for vaccinated individuals. In this case, we assume $t_{r}=1$. At $t=1$, the patient zero infects one of its neighbors in network $A$ and the other becomes vaccinated. Since this infected patient is present in both networks, it can also spread the disease in network $B$. The red lines indicate the direction of the branches of infection. In the next time step, $t=2$, this patient zero recover in both networks and the newly infected individuals continue to spread the disease. The process continues until the fourth temporary step, which is the steady state, where there are no more infected individuals that can continue spreading the disease.

\section{A. Model}

At the initial stage of the susceptible-infected-vaccinatedrecovered model (SI-R/V) all individuals in both networks are susceptible. We randomly infect an individual in network $A$, which we call patient zero, and also its counterpart in network $B$, in case it belongs to both networks. Then, all the neighbors of this patient zero (in both networks, $A$ and $B$ ) will be vaccinated and hence immunized with probability $\omega$. For the sake of simplicity, we consider that this probability is the same for all individuals. On the other hand, those neighbors who did not get vaccinated will be infected with probability $(1-\omega) \beta$. Once an individual receive a vaccine, it can no longer acquire the disease and becomes immunized. Infected individuals will recover after $t_{r}$ time steps and cannot be affected by the disease anymore. In Fig. 1 we illustrate the dynamic of our model for $t_{r}=1$ and $q=0.7$.

We illustrate the model in Fig. 1. At the beginning of the dynamic, at $t=1$, there is only one infected node (patient zero in red). In network $A$, this individual has two neighbors: One of them gets vaccinated with probability $\omega$, and the other one becomes infected with probability $(1-\omega) \beta$. As both individuals are present in both networks, their counterparts in network $B$ are also vaccinated and infected, respectively. At $t=2$, patient zero recovers in both networks and the newly infected individuals will try to infect their susceptible neighbors. In network $A$, one of the newly infected individuals has only one vaccinated neighbor and thus cannot spread the disease, and the other has three susceptible neighbors: One gets vaccinated, the other becomes infected, as well as the counterpart in network $B$, while the last remains susceptible. In network $B$ there are two infected individuals, of which only one manages to infect one of its neighbors. At the next time step, this infected individual recovers and the newly infected individuals continue to spread the disease. The dynamic continues until the system reaches a steady state in which there are no more infected nodes $(t=4)$ and the epidemic process ends.

\section{B. Theory}

In our model of the SI-R/V process we assume that the transmissibility is the same in both networks, and thus, all the individuals in the system spread the infection with the same probability. As we mentioned earlier, each infected individual in each network can infect each one of its neighbors (with probability $\beta$ ), if they have not been vaccinated earlier [with probability $(1-\omega)]$. Thus, at each time step the probability that an infected node infects a susceptible neighbor is $(1-$ $\omega) \beta$ during a period of time $t_{r}$, after which the individual recovers. Then, the overall transmissibility $T_{\beta} \equiv T\left(\beta, t_{r}, w\right)$ is the probability that an infected individual will transmit the disease to its neighbors, which is given by

$$
\begin{aligned}
T_{\beta} & =(1-\omega) \beta \sum_{t=1}^{t_{r}}[(1-\omega)(1-\beta)]^{t-1} \\
& =\frac{1-(1-\omega)^{t_{r}}(1-\beta)^{t_{r}}}{\omega+\beta-\omega \beta}(1-\omega) \beta .
\end{aligned}
$$

We can use the generating function framework to map this process onto link percolation in a system of two coupled networks $[23,24]$, after which we can write two transcendental coupled equations for $f_{A}$ and $f_{B}$. Thus, the probability of reaching, through a random chosen edge, a node that belongs to a branch of infection that expands all over the system is

$$
\begin{aligned}
f_{A}= & (1-q)\left[1-G_{1}^{A}\left(1-T_{\beta} f_{A}\right)\right] \\
& +q\left[1-G_{1}^{A}\left(1-T_{\beta} f_{A}\right) G_{0}^{B}\left(1-T_{\beta} f_{B}\right)\right], \\
f_{B}= & (1-q)\left[1-G_{1}^{B}\left(1-T_{\beta} f_{B}\right)\right] \\
& +q\left[1-G_{1}^{B}\left(1-T_{\beta} f_{B}\right) G_{0}^{A}\left(1-T_{\beta} f_{A}\right)\right] .
\end{aligned}
$$

Here $G_{0}^{i}(x)$ and $G_{1}^{i}(x)$, with $i=A, B$ are the generating functions of the degree and the excess degree distributions for each network, respectively. On one hand, $G_{1}^{A}(x)$ and $G_{1}^{B}(x)$ represent the probability that by choosing a random edge that leads to a node of degree $k$ in one network, this branch cannot spread the disease through its remaining $k-1$ connections. On the other hand, $G_{0}^{A}(x)$ and $G_{0}^{B}(x)$ take into account the probability that if the node has a counterpart node of degree $k$ in the other layer ( $B$ or $A$ ), then the branch of infection does not spread through its $k$ links, i.e., the branch does not spread in layers $B$ and $A$.

In Eqs. (2) the first term in both equations corresponds to those branches of infection that only spread within their own network, while the second term takes into account those branches that spread through both networks.

During the dynamics, the branches of infection reaches both recovered and vaccinated nodes. The difference is that 
once the infection branch crosses a link to reach a node that has been vaccinated, this vaccinated individual cannot spread the disease. Thus, we can develop in the same way as before a transmissibility $T_{\omega} \equiv T\left(\beta, t_{r}, w\right)$ as the effective probability that a susceptible neighbor in contact with an infected node, for a period of time $t_{r}$, will be vaccinated. This transmissibility is given by

$T_{\omega}=\omega \sum_{t=1}^{t_{r}}[(1-\omega)(1-\beta)]^{t-1}=\frac{1-(1-\omega)^{t_{r}}(1-\beta)^{t_{r}}}{\omega+\beta-\omega \beta} \omega$

Therefore, in the steady state of our model, the magnitude that maps with the order parameter of link percolation is not only the fraction of recovered individuals, as in the standard SIR [18], but instead, it is the sum of vaccinated and recovered, i.e., $V+R$. This is due to the fact that the infection branches also reach those nodes that were vaccinated via a link. A susceptible individual can get vaccinated only if he or she has an infected neighbor that could infect him or her through a S-I link. To consider this event in the equations, a multiplicative factor must be added, which takes into account the probability that a susceptible node becomes infected or vaccinated if its state is altered by one of its infected neighbors.

Then, for the parameter $R$, we should consider the probability that a randomly chosen node is connected to a branch of infection through at least one of its $k$ links. Thus, the fraction of recovered individuals in each network can be written as

$$
\begin{aligned}
R_{A}= & \frac{T_{\beta}}{T_{\beta}+T_{\omega}}\left\{(1-q)\left[1-G_{0}^{A}\left(1-T_{\beta} f_{A}\right)\right]\right. \\
& \left.+q\left[1-G_{0}^{A}\left(1-T_{\beta} f_{A}\right) G_{0}^{B}\left(1-T_{\beta} f_{B}\right)\right]\right\}, \\
R_{B}= & \frac{T_{\beta}}{T_{\beta}+T_{\omega}}\left\{(1-q)\left[1-G_{0}^{B}\left(1-T_{\beta} f_{B}\right)\right]\right. \\
& \left.+q\left[1-G_{0}^{B}\left(1-T_{\beta} f_{B}\right) G_{0}^{A}\left(1-T_{\beta} f_{A}\right)\right]\right\} .
\end{aligned}
$$

And the fraction of vaccinated nodes is given by

$$
\begin{aligned}
V_{A}= & \frac{T_{\omega}}{T_{\omega}+T_{\beta}}\left\{(1-q)\left[1-G_{0}^{A}\left(1-T_{\beta} f_{A}\right)\right]\right. \\
& \left.+q\left[1-G_{0}^{A}\left(1-T_{\beta} f_{A}\right) G_{0}^{B}\left(1-T_{\beta} f_{B}\right)\right]\right\}, \\
V_{B}= & \frac{T_{\omega}}{T_{\omega}+T_{\beta}}\left\{(1-q)\left[1-G_{0}^{B}\left(1-T_{\beta} f_{B}\right)\right]\right. \\
& \left.+q\left[1-G_{0}^{B}\left(1-T_{\beta} f_{B}\right) G_{0}^{A}\left(1-T_{\beta} f_{A}\right)\right]\right\} .
\end{aligned}
$$

Then, the total fraction of recovered $(R)$ and vaccinated $(V)$ individuals in the system is given by

$$
\begin{aligned}
& R=\left(R_{A}+R_{B}-\zeta_{R}\right) /(2-q) \\
& V=\left(V_{A}+V_{B}-\zeta_{V}\right) /(2-q),
\end{aligned}
$$

where $\zeta_{R}=\frac{T_{\beta}}{T_{\omega}+T_{\beta}} q\left[1-G_{0}^{A}\left(1-T_{\beta} f_{A}\right) G_{0}^{B}\left(1-T_{\beta} f_{B}\right)\right]$ is the fraction of shared nodes that are recovered and $\zeta_{V}=$ $\frac{T_{\omega}}{T_{\beta}+T_{\omega}} q\left[1-G_{0}^{A}\left(1-T_{\beta} f_{A}\right) G_{0}^{B}\left(1-T_{\beta} f_{B}\right)\right]$ is the fraction of shared nodes that are vaccinated in the steady state.

From Eqs. (1) and (2) we can see that if we use the total transmissibility, which is the sum of $T_{\beta}$ and $T_{\omega}$, as the control parameter we lose information about the probability of vaccination, $\omega$ (see Appendix A 1). Hence, we will make use of the virulence of the diseases $\beta$ as the control parameter. To this end, we fixed $t_{r}=1$ and obtain $\beta$ by inverting Eq. (1).

\section{Simulation results}

In the simulations, we generate two uncorrelated networks, $A$ and $B$, of equal size using the Molloy-Reed algorithm [56]. We randomly overlap a fraction $q$ of nodes in network $A$ with nodes in network $B$ by a one-to-one connection. The degree distribution in each network is given by $P_{i}(k)$, with $i=A, B$ and $k_{\min } \leqslant k \leqslant k_{\max }$, where $k_{\min }$ and $k_{\max }$ are the minimum and the maximum degree that a node can have. We assume that an epidemic takes place in a single realization if the number of recovered individuals is larger than a certain value $s_{c}$. Since we consider networks of size $N=10^{5}$ we chose $s_{c}=200[57,58]$. To calculate the total number of recovered and vaccinated nodes throughout the entire system, the pair of nodes that act in both networks are counted as single nodes. For the sake of simplicity we set $t_{r}=1$.

In Fig. 2 we show the total number of recovered $(R)$ and vaccinated $(V)$ nodes as a function of $\beta$ for two different vaccination scenarios, and we vary the overlap $q$ between the layers. To see the effect of the multilayer structure we consider two different networks: layer $A$ is an Erdős-Rényi (ER) network [59], with average degree $\left\langle k_{A}\right\rangle=4, k_{\min }=0$, and $k_{\max }=40$. Nodes with a connectivity equal to $k_{A}=0$ are isolated in layer $A$ and can only be infected in layer $B$. Thus, this nodes do not play a major role in the spreading process between layers. The layer $B$ is a truncated scalefree (SF) network where $P_{B}\left(k_{i}\right) \sim k_{i}^{-\lambda_{B}} e^{-k_{i} / c}$ with $\lambda_{B}=2.5$, $k_{\min }=2, k_{\max }=\sqrt{10^{5}}$ and an exponential cutoff $c=50$ [23]. Each curve corresponds to a different value of $\omega=$ $0,0.1,0.3,0.5,0.7,0.8$ from left to right. We consider the cases $q=0.1$ [Figs. 2(a) and 2(b)], and $q=0.9$ [Figs. 2(c) and 2(d)]. Insets in Fig. 2(b) and 2(d) correspond to the maximum in the fraction of vaccinated individuals $V_{\text {peak }}$, if there is one, as a function of $\omega$.

Figure 2 shows excellent agreement between simulation results and the theoretical analysis [Eqs. (4) and (6)]. As expected, the critical threshold $\beta_{c}$ increases as $q$ decreases. Hence, for less interconnected networks a more virulent disease is needed in order to become an epidemic. For instance, for $\omega=0.8$ there is a noticeable difference in $\beta_{c}$, when $q$ is low the reach of the disease is insignificant while for $q$ close to one reaches $10 \%$ of the healthy population. On top of that, it can be seen that, qualitatively, the behavior of both magnitudes are very similar regardless the overlap.

In Figs. 2(a) and 2(c) we can see that as $\omega$ increases the total fraction of recovered nodes decreases. For high values of $\beta$, such as $\beta=1$, when $\omega=0.1$ the disease reaches $90 \%$ of the population, regardless of the overlap. But as $\omega$ increases, the immunized individuals block many of the paths that would be used by the disease to spread through the population. This causes a decrease in the probability that the disease might spread or "percolate" through an edge. Thus, as the probability of vaccination gets higher, the disease has to be more virulent to reach the entire system, which translates into an increase in the critical infection threshold $\beta_{c}$. Notice that for $\omega \gtrsim 0.8$, the disease never originates an epidemic despite its virulence. 

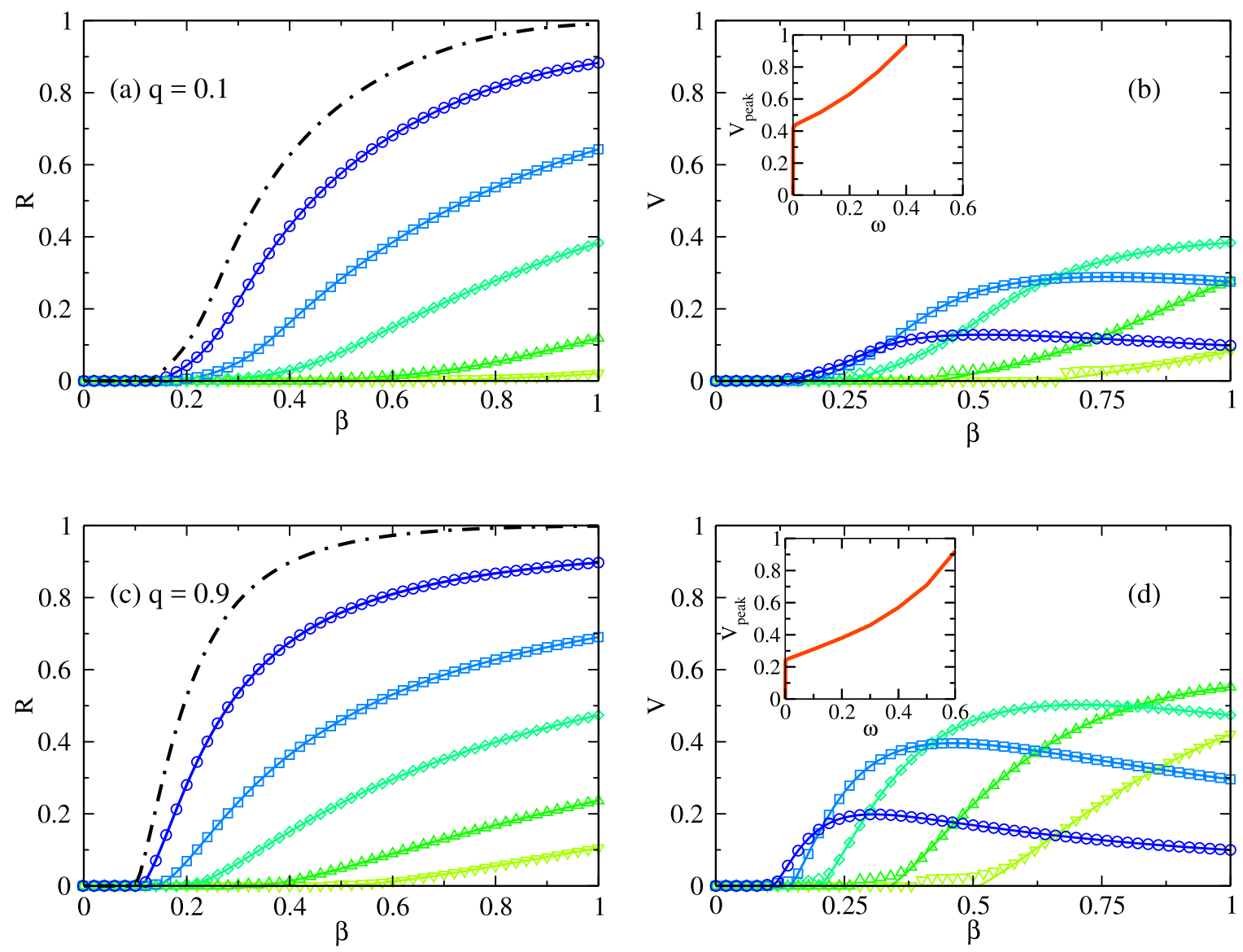

FIG. 2. Theoretical and simulation results of the total fraction of recovered $(R)$ and vaccinated $(V)$ nodes, for two different overlap scenarios $(q=0.1,0.9)$, as a function of the virulence of the diseases $\beta$ and for different values of $\omega$, in the steady state of the process. We consider $t_{r}=1$, [(a) and (b)] $q=0.1$ and [(c) and (d)] $q=0.9$, and $\omega=0,0.1,0.3,0.5,0.7,0.8$ from left to right. The symbols correspond to the simulation results and the lines correspond to the theoretical evaluation of Eqs. (6). The multiplex network consists of two layers, each of size $N=10^{5}$. Layer $A$ is an ER network with $\left\langle k_{A}\right\rangle=4$ with $k_{\min }=0$ and $k_{\max }=40$, and layer $B$ is an SF network with $\lambda_{B}=2.5, k_{\min }=2$, $k_{\max }=\sqrt{10^{5}}$ and exponential cutoff $c=50$, thus $\left\langle k_{B}\right\rangle \simeq 3.66$. Simulation results are averaged over $10^{4}$ realizations.

In contrast, in Figs. 2(b) and 2(d), we can see that the total fraction of vaccinated nodes does not behave monotonically with $\beta$. For small $\beta, V$ increases until it reaches a maximum value and then starts to decrease. This maximum value varies with $\omega$, is more pronounced for low values of $\omega$, and vanishes as $\omega$ becomes larger. The inset in each plot shows this peak as a function of $\omega$. When the probability of vaccination is equal to zero, there is no vaccinated nodes. However, slightly above $\omega=0$ a peak exhibits. Then, as $\omega$ increases the fraction of vaccinated individuals in the peak also increases, reaching a certain value of $\omega$ above which the peak vanishes. Note that this maximum value also varies with the overlap fraction, $q$.

The origin of the peaks is the competition between the spread of the disease and the vaccination process. For instance, let us consider the case of low values of $\omega$, such as $\omega=0.1$. Slightly above $\beta_{c}$, the disease is not virulent enough so increasing $\beta$ the number of infected and vaccinated nodes increases. This is true until a certain value of $\beta$ that corresponds to the peak in $V$, above which individuals are more likely to become infected rather than vaccinated. On the contrary, in the case of high $\omega$ values, the peak is not exhibited. This is due to the fact that the virulence of the disease is not strong enough to overcome the vaccination. Furthermore, as $\omega$ increases and for high values of overlap, the fraction of vaccinated individuals is much higher than for low values of $q$.

Now, in order to study the interplay between the layers, we vary the overlap in the system and consider a scenario of low and high probability of vaccination, $\omega=0.1$ (left) and $\omega=0.7$ (right). We show in Fig. 3 the difference between the total number of recovered individuals $\left(R_{A}-R_{B}\right)$ of each layer as a function of $\beta$. Each curve corresponds to different values of $q$, with $q \in[0,1]$, and $\Delta q=0.1$. In Figs. 3(a) and 3(b) we consider in layer $A$ an ER network with average degree $\left\langle k_{A}\right\rangle=4, k_{\min }=0$ and $k_{\max }=40$, and in layer $B$ a SF network with $\lambda_{A}=\lambda_{B}=2.5, k_{\min }=2$ and $k_{\max }=\sqrt{10^{5}}$, and an exponential cutoff $c=135$, thus $\left\langle k_{B}\right\rangle \simeq 4$ [23]. Note that this type of SF network is appropriate to describe scenarios and structures of real-word systems [60]. For the case of isolated networks, $q=0$, we can observe that there is a notable difference between the fraction of recovered individuals in each layer. For low values of $\beta$ it is well known that the level of the epidemic is higher in layer $B$ due to the ultrasmall-world property [61]. In Fig. 3(a), when the probability of vaccination is low, there are two regimes: for low values of $\beta$, the disease spreads more in the SF network, but above 

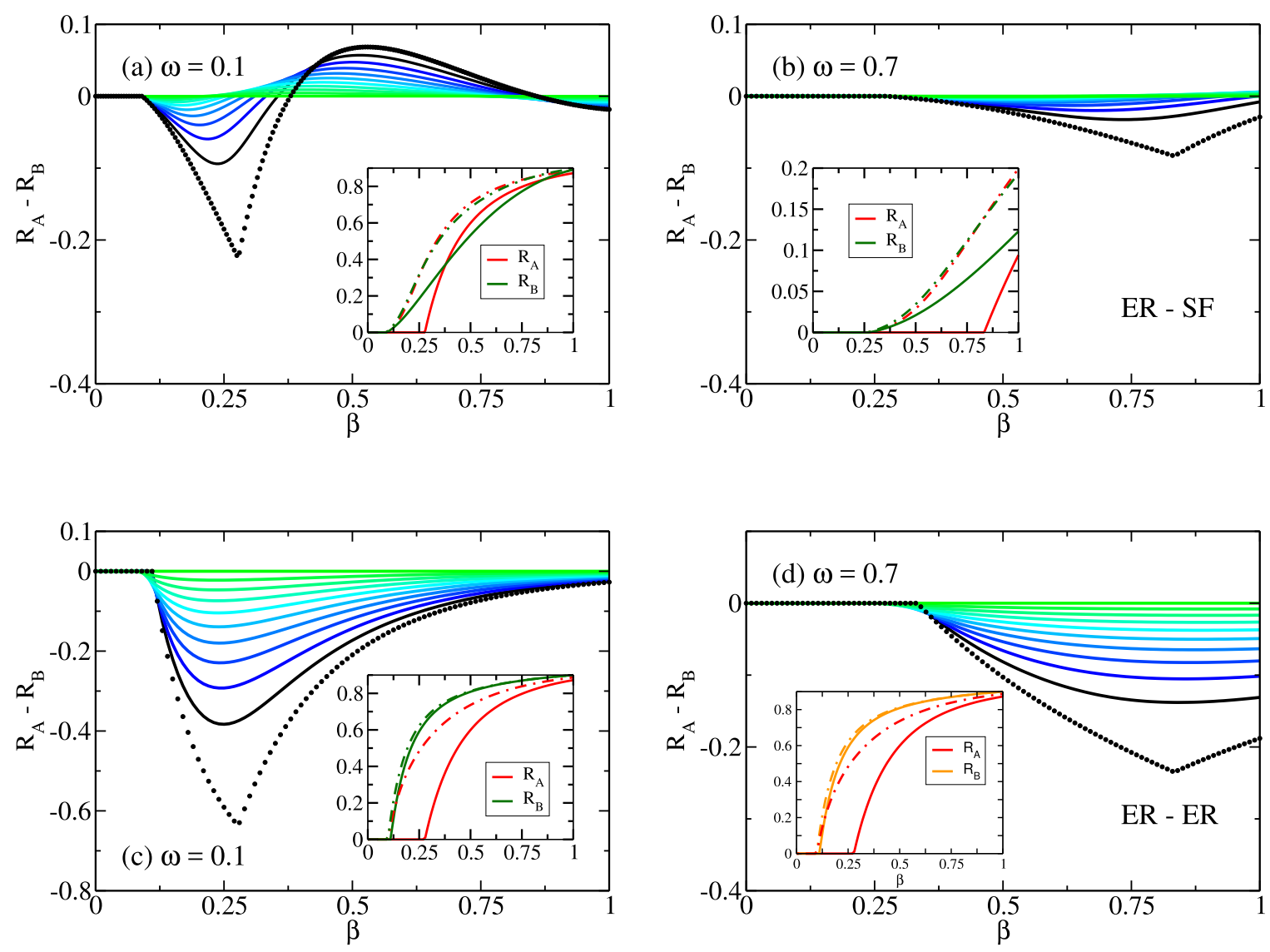

FIG. 3. Difference between the fraction of recovered and vaccinated individuals in both layers as a function of the virulence of the disease for different values of the overlap $q$. We consider $\omega=0.1$ (left), 0.7 (right), and $t_{r}=1$. The overlap $q$ varies from 0 (๑) to 1 ( - ) with $\Delta q=0.1$. The curves correspond to the theoretical evaluation of Eqs. (6). In (a) and (b) we consider in layer $A$ an ER network with average degree $\left\langle k_{A}\right\rangle=4, k_{\min }=0$ and $k_{\max }=40$, and in layer $B$ a SF network with $\lambda_{A}=\lambda_{B}=2.5, k_{\min }=2$ and $k_{\max }=\sqrt{10^{5}}$, and exponential cutoff $c=135$, thus $\left\langle k_{B}\right\rangle \simeq 4$. Both networks are of the same size $N=10^{5}$. In (c) and (d) the system is composed by two ER, layer $A$ with $\left\langle k_{A}\right\rangle=4$ and layer $B$ with $\left\langle k_{B}\right\rangle=10$, both with $k_{\min }=0$ and $k_{\max }=40$. The insets show the fraction of recovered individuals in each layer for the case of $q=0$ (solid lines) and $q=0.5$ (dash-dotted lines).

a certain $\beta$ value the ER network topology is more efficient to propagate the infection. In Fig. 3(c), the networks have the same topology, and thus, it is expected that there will be more recovered individuals in the network (layer $B$ ) with larger average degree.

In Fig. 3(b) we can observe that for $\omega=0.7$ the vaccination strategy is very effective, and the difference between the spread of disease is low. As we can see from the inset, this is due to the fact that the disease almost does not spread. Besides, when $q=0$ (solid lines), the critical value above which the epidemic occurs is different for each network and much lower for the SF network. But for the case of $q=0.5$ (dash-dotted lines) this value is dominated by the network where the epidemic spreads more easily. Besides, for $\beta=1$ the infection reaches nearly $10 \%$ of the population and even more in the SF network. However, when the networks are overlapped the infection spreads more easily, and hence as $q$ increases the difference between the $R_{A}$ and $R_{B}$ shrinks to zero.

Focusing on the critical threshold $\beta_{c}$, this can be obtained theoretically from the intersection of the Eqs. (2) where all branches of infection stop spreading, which is given when $f_{A}=f_{B}=0$. This is equivalent to find the solution of the system $\operatorname{det}(J-I)=0$, where $I$ is the identity matrix and $J$ is the Jacobian matrix of the coupled equation with $\left.J_{i, k}\right|_{f_{i}=f_{k}=0}=\partial f_{i} /\left.\partial f_{k}\right|_{f_{i}=f_{k}=0}$. In Ref. [44], this theoretical critical value has been obtained for the standard SIR model in a partially overlapped multiplex network, $T_{c}^{\mathrm{SIR}}$, and it is equal to the transmissibility of our model, $T_{c}^{\mathrm{SIR}}=T_{c}$. However, the critical infection probabilities are different in both models. For instance, for $t_{r}=1$ it is well known that $T_{c}^{\mathrm{SIR}}=\beta_{c}^{\mathrm{SIR}}$, and in our model $T_{c}=\beta_{c}(1-\omega)$. Thus $\beta_{c}^{\mathrm{SIR}}=\beta_{c}(1-\omega)$. Next, to account for how the magnitudes change with $\omega$, we will focus on the critical value of virulence of the diseases, $\beta_{c}=\beta_{c}^{\mathrm{SIR}} /(1-\omega)$, and not on the critical transmissibility. Using numerical evaluations we find a physical and stable solution for $\beta_{c}$.

In Fig. 4 we display a surface plot that shows how the critical infection probability $\beta_{c}$ depends on the vaccination probability $\omega$ and the overlapping between layers $q$. In the figures at the top, we consider the cases of an ER network in layer $A$ and an SF network in layer $B$. Figures at the bottom correspond to the case of two ER networks. In all figures we set $t_{r}=1$.

From Fig. 4 we can see that the surface, which represents the critical virulence, separates into two phases: epidemic 

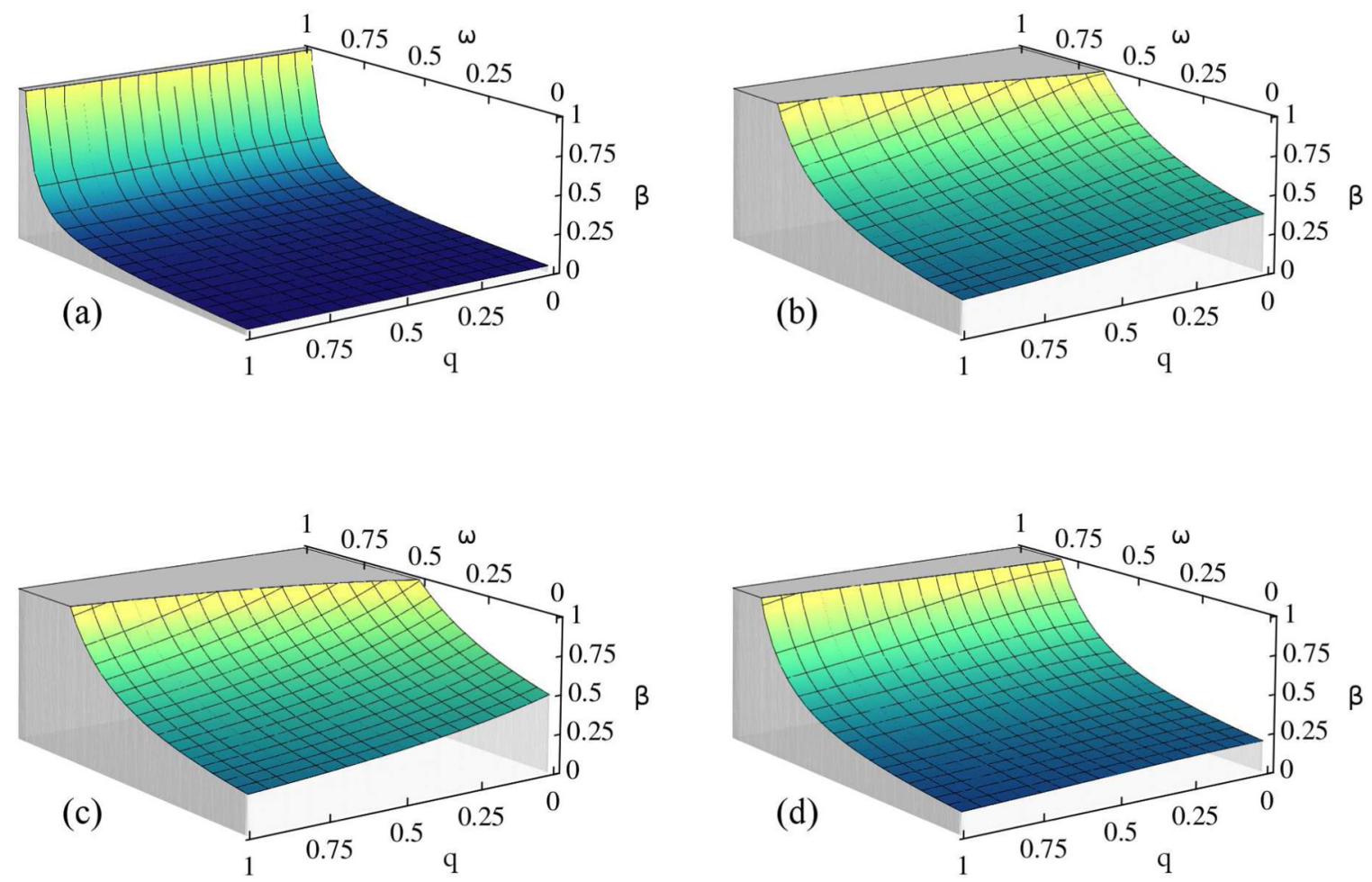

FIG. 4. Surface phase diagram obtained theoretically for the case of $t_{r}=1$. In the top panels we consider in layer $A$ an ER network with $\left\langle k_{A}\right\rangle=2$ with $k_{\min }=0$ and $k_{\max }=40$, and in layer $B$ a SF network with (a) $\lambda_{B}=1.5$ and (b) $\lambda_{B}=3.5, k_{\min }=2, k_{\max }=\sqrt{10^{5}}$ and exponential cutoff $c=135$. On the bottom we consider two ER networks with $\left\langle k_{A}\right\rangle=2$ in layer $A$ and (c) $\left\langle k_{B}\right\rangle=2$ (d) $\left\langle k_{B}\right\rangle=5$ in layer $B$. In all $k_{\text {min }}=0$ and $k_{\max }=40$. In all networks, $N=10^{5}$. We can see that there are two well marked regions: an epidemic and a nonepidemic phase, separated by a critical value of $\beta$. Increasing the overlapping $q$ facilitates the spread of the epidemic, thus, $\beta_{c}$ decreases and the epidemic phase regime is smaller. In all figures there is a critical value of $\omega$ above which, regardless of the virulence of the disease, there is no epidemic. In the case of no vaccination, $\omega=0$, we recover the regular SIR in multiplex networks and as $\omega$ increases, there will be more vaccinated individuals so $\beta_{c}$ increases.

(regime above the surface) and epidemic free (regime below the surface). We observe that in all cases as $q$ decreases and $\omega$ increases the nonepidemic phase becomes wider. Furthermore, as we increase $\omega$, for all values of $q$, we can see the existence of a threshold above which, even for very virulent diseases, the outbreak will never become an epidemic. The behavior of the plots in Fig. 4 is similar. From the figures above, we can see that by decreasing $\lambda_{B}$, we increase the heterogeneity of the network, and this facilitates the spread of the disease, since once the infection reaches a hub, a high degree node, it spreads rapidly across the network. The same applies for figures on the bottom: By connecting two networks with low average degree, the infection does not propagate so easily.

Finally, in Fig. 5 we theoretically compare our strategy of dynamic vaccination with a very effective strategy, targeted vaccination [62], and with another that usually performs poorly, random vaccination [63]. In the case of random vaccination, a random fraction $V$ of nodes in layer $A$ are vaccinated before the spreading of the disease, and if those nodes have a counterpart in layer $B$, they will also get immunization against the infection. On the other hand, in the targeted immunization strategy, we choose a fraction $V$ of the highest connected individuals in layer $A$ to get vaccinated.

In [Figs. 5(a) and 5(b)] we considered the case of two ER multiplex networks with the same average degree, $\left\langle k_{A}\right\rangle=$ $\left\langle k_{B}\right\rangle=5$, and in [Figs. 5(c) and 5(d)] we considered two scale-free networks with $\lambda_{A}=\lambda_{B}=2.5$. We set $t_{r}=1, \beta=$ 0.3 , and vary the overlap between networks [Figs. 5(a) and 5(c)] $q=0.3$ and [Figs. 5(c) and 5(d)] $q=0.7$. Figure 5 shows the total fraction of recovered nodes in the steady state as a function of $V$ and $\omega$. In Fig. 5(a), when the total fraction of vaccinated individuals is, for instance, $V=0.2$, for the case of random vaccination $R \simeq 0.55$, while targeted immunization performs much better, $R \simeq 0.15$. On the other hand, note that for dynamic vaccination there are two possible outcomes of $R$ depending on $\omega$. Using the same amount of vaccines $(V=0.2)$, if $\omega \simeq 0.3$, then $R \simeq 0.4$, but for $\omega \simeq$ 0.6 , we have a much more interesting scenario where the final fraction of recovered individuals is close $R \simeq 0.1$. In this last case, there is almost no difference between targeted and dynamic vaccination. In some cases, there is a threshold value $V^{*}$, above which there is no epidemic. For instance, in Fig. 5(b), $V^{*}=0.45$ (targeted) and $V^{*}=0.65$ (random). However, in the case of dynamic vaccination $V^{*} \rightarrow 0$ when $\omega \rightarrow 1$, i.e., a very small amount of vaccines is needed to prevent the epidemic. In short, from Fig. 5 we can see that, for the same amount of vaccinated individuals, the number of recovered individuals is significantly lower for dynamic vaccination compared to random vaccination. This is observed in all plots, which indicates that our strategy is significantly more effective than random vaccination. Especially, in more 

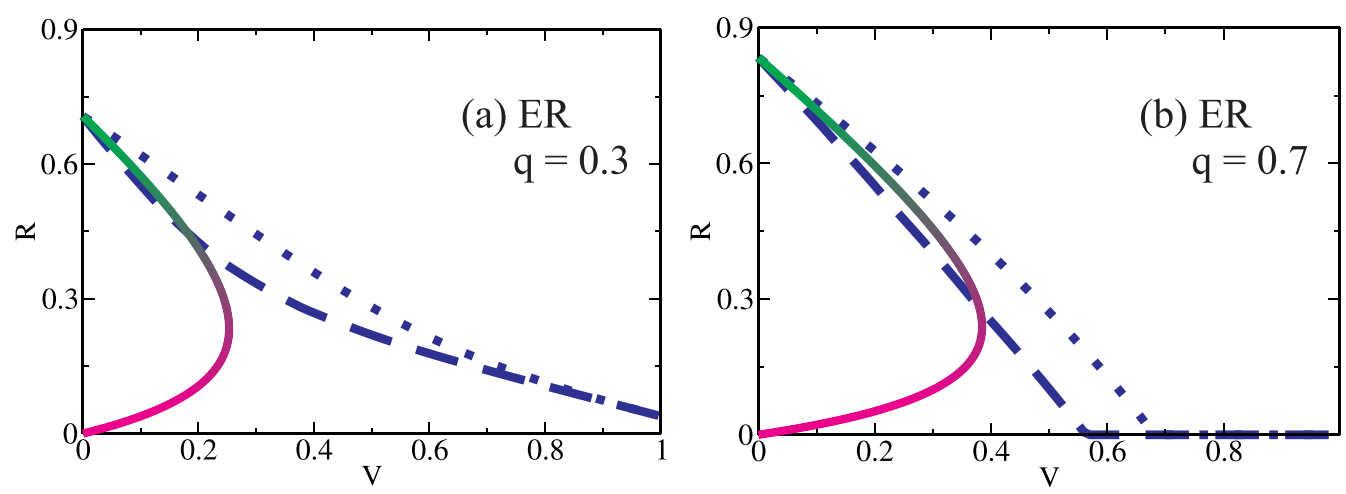

$\omega$
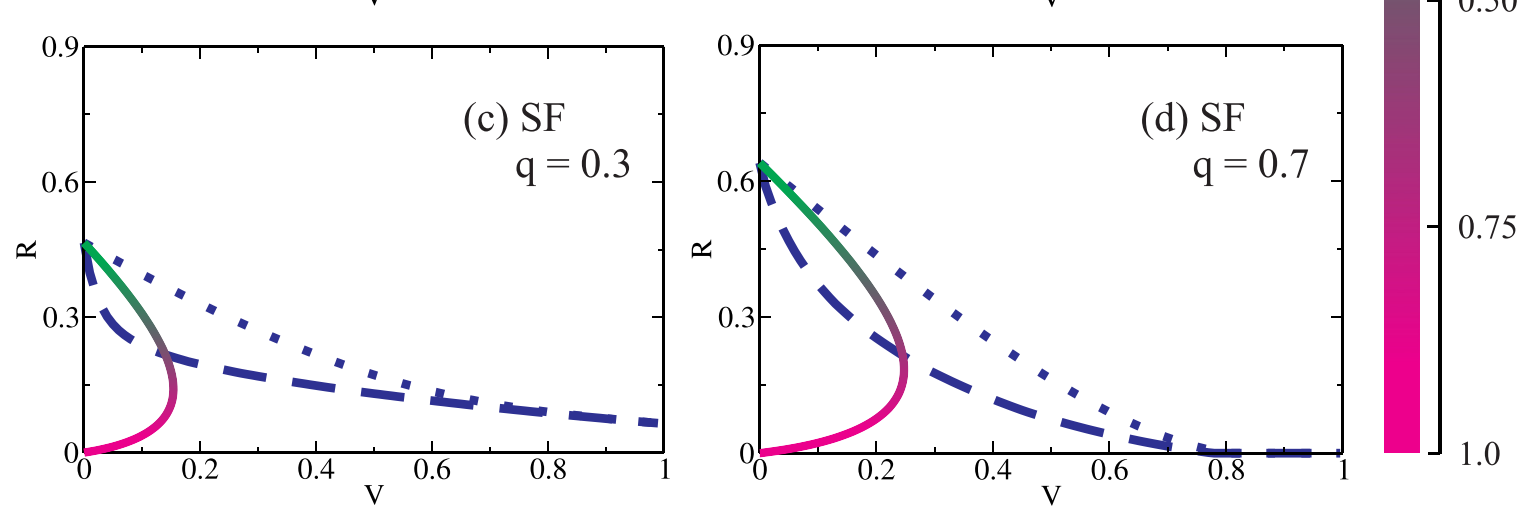

FIG. 5. Comparison among dynamic, random, and targeted vaccination. Theoretical results of the total fraction of recovered (R) nodes as a function of V. All dotted lines correspond to random vaccination (....), the dashed lines to targeted immunization ( $-1 \cdot)$, and the colored solid lines correspond to our strategy of dynamic vaccination. For the case of dynamic vaccination the colors in the curves indicate different values of $\omega$. Notice that $\omega$ decreases from top to bottom. We set $t_{r}=1, \beta=0.3$ and consider different values of overlap [(a)-(c)] $q=0.3$ and [(b)-(d)] $q=0.7$. In (a) and (b) we used two ER networks with $\left\langle k_{A}\right\rangle=\left\langle k_{B}\right\rangle=5$ with $k_{\min }=0$ and $k_{\max }=40$, and in (c) and (d) two scale-free networks, with $\lambda_{A}=\lambda_{B}=2.5, k_{\min }=2, k_{\max }=\sqrt{N}, N=10^{5}$, and exponential cutoff $c=50$.

heterogeneous networks, such as SF networks, we can see that this strategy is even more efficient.

On the other hand, if we compare our strategy with targeted vaccination, then there is a certain value of $\omega$ above which in dynamic vaccination performs much better, even using a lower amount of vaccines than in targeted vaccination. Notably, this is also observed for scale-free networks, in which the immunization of the hubs is usually an extremely effective strategy. This is because when the contacts of an infected node are immunized, it is highly likely that at least one of that contacts is a hub. Thus, for SF networks our strategy also targets high connectivity nodes, preventing a massive spreading of the disease.

Furthermore, when the system is more interconnected, the disease spreads more. Therefore, more vaccines are needed to prevented it from becoming an epidemic. For example, for a final fraction of recovered individuals equal to $20 \%$ of the population, it is required to vaccinate a fraction of individuals equal to $V(q=0.3) \simeq 0.25$ and $V(q=0.7) \simeq 0.4$.

To summarize, we demonstrate that, on top of a multiplex network structure, dynamic vaccination strategy is much more efficient than random immunization. Using the same amount of vaccines, the total fraction of recovered individuals is always lower in the case of dynamic vaccination. Besides, in our strategy, depending on the parameters, we obtain a region where, regardless of the virulence of the disease, it will never become an epidemic. In comparison, for the same $V$, the fraction of recovered individuals in dynamic vaccination will always be lower than in the case of random immunization. Thus, our strategy can be more beneficial to be implemented in real scenarios, such as in outbreaks of Ebola or influenza.

\section{DISCUSSION}

In summary, we studied a model of dynamic vaccination on a system composed of two partially overlapped networks, where $q$ is the fraction of common nodes in both networks. In our model, susceptible individuals in contact with infected patients have the opportunity to be vaccinated before the their neighbors attempt to infect them. That is, each time an infected person comes into contact with a susceptible individual, this one will try to get vaccinated with probability $\omega$ and if he does not succeed he will be infected with probability $\beta$. Each infected node is assumed to recover after $t_{r}$ time steps and will become immunized. Besides, vaccinated nodes are also immunized and cannot be infected or infect others. By mapping this process into bond percolation and using the framework of generating functions, we analyzed analytically the total fraction of recovered and vaccinated nodes in the steady state as a function of the virulence of the disease $\beta$ for different values of $\omega$ and $q$, and we found a perfect agreement between the theoretical and the simulation results. As expected we find that as $\omega$ increases, the epidemic threshold $\beta_{c}$ becomes larger and disappears for very large values of $\omega$. We also find 
a peak, for certain values of the parameters, in the fraction of vaccinated nodes as a function of $\omega$, which is determined by the competition between the vaccination strategy and the spread of disease. We find an interesting phase diagram in the plane $\beta-\omega$, where we can see an epidemic phase, which diminishes as $q$ and $\omega$ increase, and a nonepidemic phase, where the diseases cannot spread. A remarkable result of the phase diagram is that for certain values of $q$ and $\omega$, regardless of the virulence of the disease, it will never become an epidemic. Finally, we demonstrate that our strategy is always more efficient than random immunization and, depending on $\omega$, performs better or worse than targeted vaccination. For high vaccination probabilities, i.e., when the population is more receptive to be vaccinated, dynamic immunization is the most effective strategy to avoid or mitigate an epidemic. This goal can be achieved using efficiently the available vaccines, immunizing a small fraction of the population and creating a barrier of vaccinated individuals that the infection cannot pass through.

\section{ACKNOWLEDGMENTS}

S.H. thanks the Israel Science Foundation, ONR, ARO, the Israel Ministry of Science and Technology (MOST) with the Italy Ministry of Foreign Affairs, BSF-NSF, MOST with the Japan Science and Technology Agency, the BIU Center for Research in Applied Cryptography and Cyber Security, and DTRA (Grant No. HDTRA-1-10-1- 0014) for financial support. L.G.A.Z., M.A.D., and L.A.B. wish to thank to UNMdP, FONCyT, and CONICET (Pict 0429/2013, Pict 1407/2014, and PIP 11220130100443) for financial support.

\section{APPENDIX}

\section{Dependence on $T_{\beta}$}

In this section we show the dependence on the overall transmissibility $T_{\beta}$ of the recovered and vaccinated fraction of nodes, $R$ and $V$, in the steady state. Also, we show the dependence on the prefactors that multiply Eqs. (4) and (5), which measure the relative weight of the probabilities of infection (or vaccination) in the dynamic.

As we saw earlier in Sec. II B, from Eqs. (1) and (3) we find the prefactors in Eqs. (4) and (5) as

$$
\begin{aligned}
& B_{\beta}=\frac{T_{\beta}}{T_{\beta}+T_{\omega}}=\frac{(1-\omega) \beta}{(1-\omega) \beta+\omega}, \\
& B_{\omega}=\frac{T_{\omega}}{T_{\beta}+T_{\omega}}=\frac{\omega}{(1-\omega) \beta+\omega} .
\end{aligned}
$$

Note, interestingly, that these prefactors are independent of the recovery time $t_{r}$.

In Fig. 6(a) we plot the total fraction of recovered nodes $R$, (b) $R / B_{\beta}$, (c) total fraction of vaccinated nodes $V$, and (d) $V / B_{\omega}$, as a function of the epidemic transmissibility $T_{\beta}$ for different values of $\omega$, from $\omega=0.1$ to $\omega=0.8$, with intervals of $\Delta \omega=0.1$. For the sake of simplicity we consider the case $t_{r}=1$. Thus, from Eq. (1) the epidemic transmissibility is reduced to $T_{\beta}=(1-\omega) \beta$.
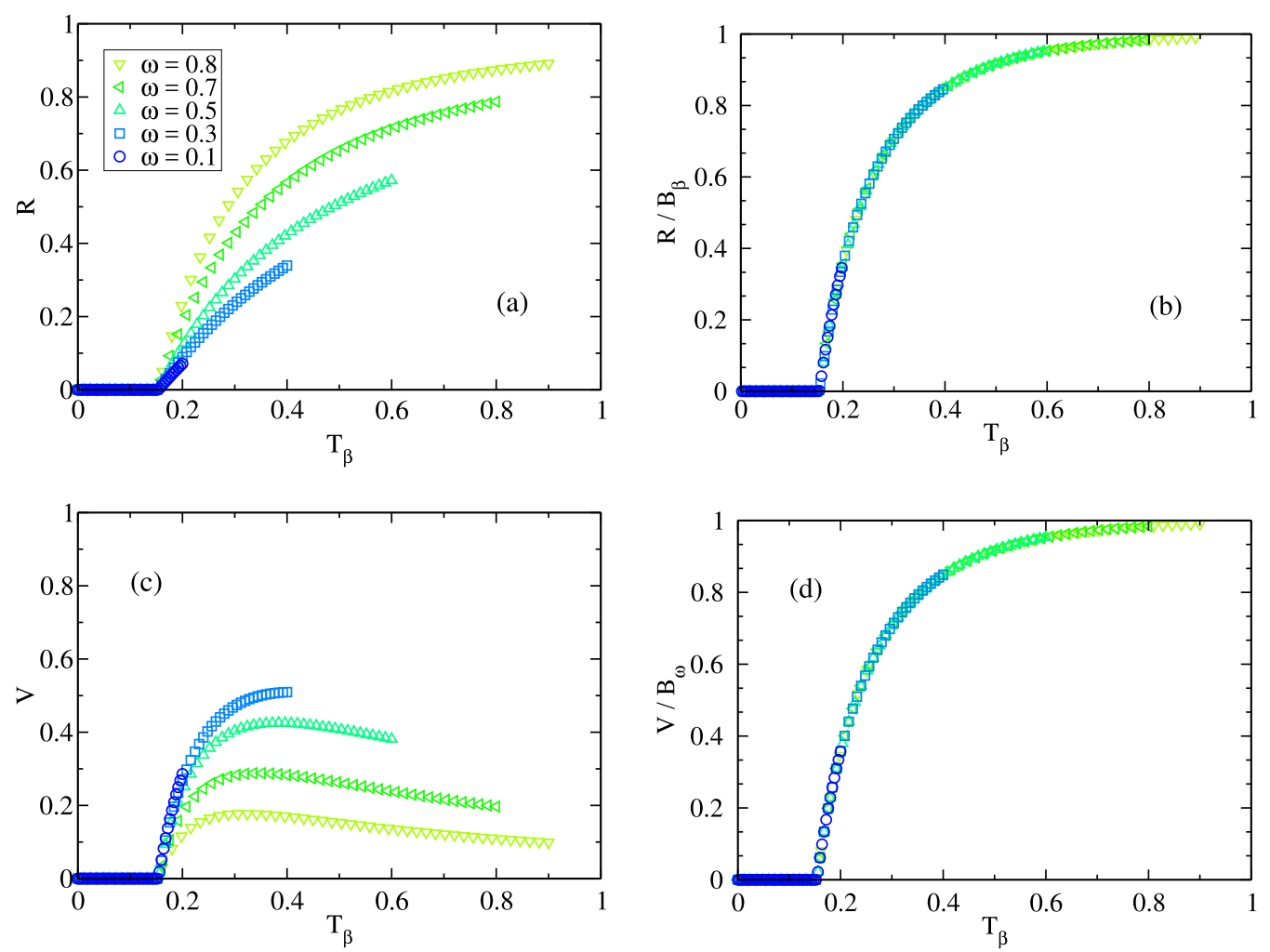

FIG. 6. Theoretical results of the total fraction of (a) recovered $R$, (b) $R / B_{\beta}$, (c) vaccinated $V$, and (d) $V / B_{\omega}$ nodes as a function of the overall transmissibility $T_{\beta}=(1-\omega) \beta$ when $t_{r}=1$ and for different values of $\omega$ in the steady state. The multiplex network consists of two ER networks and we set $q=0.3$. Each network size is $N=10^{5}$ and $\left\langle k_{A}\right\rangle=\left\langle k_{B}\right\rangle=5$ with $k_{\min }=0$ and $k_{\max }=40$. 
The multiplex network consists of two coupled ER networks with $q=0.3$, average degree $\left\langle k_{A}\right\rangle=\left\langle k_{B}\right\rangle=5$, and $k_{\min }=0$ and $k_{\max }=40$.

As we can see, in Figs. 6(a) and 6(c) for the different $\omega$ values, when $R$ and $V$ are plotted as a function of $T_{\beta}$ all the curves collapse in the same critical threshold, when $\omega=0$ [62], which is equal to

$$
\left.T_{\beta}\right|_{\left(\beta=\beta_{c}\right)}=\frac{1}{\langle k\rangle(1+q)} .
$$

Since we consider $\langle k\rangle=\left\langle k_{A}\right\rangle=\left\langle k_{B}\right\rangle=5$ and $q=0.3$, then for Fig. 6 the critical threshold is $\left.T_{\beta}\right|_{\left(\beta=\beta_{c}\right)} \simeq 0.15$.
Notice that, for instance, for $t_{r}=1$ we have $T_{\beta}=\beta$ (1$\omega)$, and thus for a fixed vaccination probability $T_{\beta} \in[0,1-$ $\omega]$, with $\omega \in[0,1]$. Then, $T_{\beta} \notin[0,1]$, as the transmissibility in the standard SIR. In Fig. 6, as each curve corresponds to a different value of $\omega$, they will reach maximum values for different $T_{\beta}$. Furthermore, as $\omega$ increases, each curve becomes shorter since the dependency on $\omega$ is still present in the prefactors.

In Figs. 6(b) and 6(d) we multiply all the curves (fraction of recovered nodes) by the weighted scaled prefactor of infection, and we can see that all data collapse into a single curve. This is due to the fact that with this scaling the dependence on $\omega$ is removed.
[1] C. Fraser, C. A. Donnelly, S. Cauchemez, W. P. Hanage, M. D. Van Kerkhove, T. D. Hollingsworth, J. Griffin, R. F. Baggaley, H. E. Jenkins, E. J. Lyons et al., Science 324, 1557 (2009).

[2] B. Pourbohloul, A. Ahued, B. Davoudi, R. Meza, L. A. Meyers, D. M. Skowronski, I. Villaseñor, F. Galván, P. Cravioto, D. J. Earn et al., Influenza Respir. Virus. 3, 215 (2009).

[3] L. F. White, J. Wallinga, L. Finelli, C. Reed, S. Riley, M. Lipsitch, and M. Pagano, Influenza Respir. Virus. 3, 267 (2009).

[4] S. Riley, C. Fraser, C. A. Donnelly, A. C. Ghani, L. J. AbuRaddad, A. J. Hedley, G. M. Leung, L.-M. Ho, T.-H. Lam, T. Q. Thach et al., Science 300, 1961 (2003).

[5] L. A. Meyers, B. Pourbohloul, M. E. Newman, D. M. Skowronski, and R. C. Brunham, J. Theor. Biol. 232, 71 (2005).

[6] V. Colizza, A. Barrat, M. Barthélemy, and A. Vespignani, BMC Med. 5, 34 (2007).

[7] P. Bajardi, C. Poletto, J. J. Ramasco, M. Tizzoni, V. Colizza, and A. Vespignani, PLOS ONE 6, e16591 (2011).

[8] R. M. Anderson and R. M. May, Infectious Diseases of Humans: Dynamics and Control (Oxford University Press, Oxford, 1992).

[9] N. T. J. Bailey, The Mathematical Theory of Infectious Diseases (Griffin, London, 1975).

[10] C. Cattuto, W. V. den Broeck, A. Barrat, V. Colizza, J.-F. Pinton, and A. Vespignani, PLoS ONE 5, e11596 (2010).

[11] M. C. Gonzalez, C. A. Hidalgo, and A.-L. Barabasi, Nature 453, 779 (2008).

[12] J. Gómez-Gardeñes, V. Latora, Y. Moreno, and E. Profumo, Proc. Natl. Acad. Sci. USA 105, 1399 (2008).

[13] S. Boccaletti, V. Latora, Y. Moreno, M. Chavez, and D. Hwang, Phys. Rep. 424, 175 (2006).

[14] R. Cohen and S. Havlin, Complex Networks: Structure, Robustness and Function (Cambridge University Press, Cambridge, 2010).

[15] A. Barrat, M. Barthélemy, R. Pastor-Satorras, and A. Vespignani, Proc. Natl. Acad. Sci. USA 101, 3747 (2004).

[16] M. E. J. Newman, Networks: An Introduction (Oxford University Press, Oxford, 2010).

[17] R. Pastor-Satorras and A. Vespignani, Phys. Rev. Lett. 86, 3200 (2001).

[18] M. E. J. Newman, Phys. Rev. E 66, 016128 (2002).

[19] Y. Moreno, R. Pastor-Satorras, and A. Vespignani, Eur. Phys. J. B 26, 521 (2002).

[20] L. D. Valdez, P. A. Macri, and L. A. Braunstein, PLOS ONE 7, e44188 (2012).
[21] D. S. Callaway, M. E. J. Newman, S. H. Strogatz, and D. J. Watts, Phys. Rev. Lett. 85, 5468 (2000).

[22] R. Cohen, S. Havlin, and D. ben Avraham, Handbook of Graphs and Networks (Wiley-VCH, Berlin, 2002).

[23] M. E. J. Newman, S. H. Strogatz, and D. J. Watts, Phys. Rev. E 64, 026118 (2001).

[24] L. A. Braunstein, Z. Wu, Y. Chen, S. V. Buldyrev, T. Kalisky, S. Sreenivasan, R. Cohen, E. López, S. Havlin, and H. E. Stanley, Int. J. Bifurcat. Chaos 17, 2215 (2007).

[25] C. Castellano and R. Pastor-Satorras, Phys. Rev. Lett. 105, 218701 (2010).

[26] R. Pastor-Satorras, C. Castellano, P. Van Mieghem, and A. Vespignani, Rev. Mod. Phys. 87, 925 (2015).

[27] M. De Domenico, C. Granell, M. A. Porter, and A. Arenas, Nat. Phys. 12, 901 (2016).

[28] W. Wang, M. Tang, H. E. Stanley, and L. A. Braunstein, Rep. Prog. Phys. 80, 036603 (2017).

[29] C. Buono, F. Vazquez, P. A. Macri, and L. A. Braunstein, Phys. Rev. E 88, 022813 (2013).

[30] C. Granell, S. Gómez, and A. Arenas, Phys. Rev. Lett. 111, 128701 (2013).

[31] E. Cozzo, R. A. Baños, S. Meloni, and Y. Moreno, Phys. Rev. E 88, 050801(R) (2013).

[32] J. Sanz, C.-Y. Xia, S. Meloni, and Y. Moreno, Phys. Rev. X 4, 041005 (2014).

[33] C. Lagorio, M. Dickison, F. Vazquez, L. A. Braunstein, P. A. Macri, M. V. Migueles, S. Havlin, and H. E. Stanley, Phys. Rev. E 83, 026102 (2011).

[34] Z. Wang, M. A. Andrews, Z.-X. Wu, L. Wang, and C. T. Bauch, Phys. Life Rev. 15, 1 (2015).

[35] L. Valdez, P. Macri, and L. Braunstein, Physica A 392, 4172 (2013).

[36] L. Wang, Y. Zhang, T. Huang, and X. Li, Phys. Rev. E 86, 032901 (2012).

[37] Z. Wang, C. T. Bauch, S. Bhattacharyya, A. d'Onofrio, P. Manfredi, M. Perc, N. Perra, M. Salathé, and D. Zhao, Phys. Rep. 664, 1 (2016).

[38] R. Pastor-Satorras and A. Vespignani, Phys. Rev. E 65, 036104 (2002).

[39] N. Madar, T. Kalisky, R. Cohen, D. ben Avraham, and S. Havlin, Eur. Phys. J. B 38, 269 (2004).

[40] R. Cohen, S. Havlin, and D. ben-Avraham, Phys. Rev. Lett. 91, 247901 (2003). 
[41] M. Dickison, S. Havlin, and H. E. Stanley, Phys. Rev. E 85, 066109 (2012).

[42] A. Saumell-Mendiola, M. Á. Serrano, and M. Boguñá, Phys. Rev. E 86, 026106 (2012).

[43] O. Yagan, D. Qian, J. Zhang, and D. Cochran, IEEE JSAC 31, 1038 (2013).

[44] C. Buono, L. G. Alvarez-Zuzek, L. A. Braunstein, and P. A. Macri, PLOS ONE 9, e92200 (2014).

[45] G. F. de Arruda, E. Cozzo, T. P. Peixoto, F. A. Rodrigues, and Y. Moreno, Phys. Rev. X 7, 011014 (2017).

[46] M. Tizzoni, P. Bajardi, C. Poletto, J. J. Ramasco, D. Balcan, B. Gonçalves, N. Perra, V. Colizza, and A. Vespignani, BMC Med. 10, 165 (2012).

[47] L. Valdez, R. H. Aragão, H. Stanley, and L. Braunstein, Sci. Rep. 5, 12172 (2014).

[48] M. F. C. Gomes, A. P. y Piontti, L. Rossi, D. Chao, I. Longini, M. E. Halloran, and A. Vespignani, PLOS Curr. 6 (2014).

[49] L. G. Alvarez-Zuzek, H. E. Stanley, and L. A. Braunstein, Sci. Rep. 5, 12151 (2015).

[50] Z. Wang, D.-W. Zhao, L. Wang, G.-Q. Sun, and Z. Jin, Europhys. Lett. 112, 48002 (2015).

[51] F. Fenner, D. A. Henderson, I. Arita, Z. Jezek, and I. D. Ladnyi, Smallpox and Its Eradication (World Health Organization, Geneva, 1988), pp. 343-345.
[52] A. Perisic and C. T. Bauch, PLoS Comput. Biol. 5, e1000280 (2009).

[53] J. Müller, B. Schönfisch, and M. Kirkilionis, J. Math. Biol. 41, 143 (2000).

[54] S. Merler, M. Ajelli, L. Fumanelli, S. Parlamento, A. P. y Piontti, N. E. Dean, G. Putoto, D. Carraro, I. M. Longini, Jr., M. E. Halloran et al., PLoS Neglect. Trop. Dis. 10, e0005093 (2016).

[55] P.-S. Gsell, A. Camacho, A. J Kucharski, C. H Watson, A. Bagayoko, S. Danmadji Nadlaou et al., Lancet Infect. Dis. 17, 1276 (2017).

[56] M. Molloy and B. Reed, Rand. Struct. Algor. 6, 161 (1995).

[57] C. Lagorio, M. V. Migueles, L. A. Braunstein, E. López, and P. A. Macri, Physica A 388, 755 (2009).

[58] Y. Hu, S. Ji, Y. Jin, L. Feng, H. E. Stanley, and S. Havlin, Proc. Natl. Acad. Sci. USA 115, 7468 (2018).

[59] P. Erdos and A. Rényi, Publ. Math. Inst. Hung. Acad. Sci. 5, 17 (1960).

[60] L. A. N. Amaral, A. Scala, M. Barthélemy, and H. E. Stanley, Proc. Natl. Acad. Sci. USA 97, 11149 (2000).

[61] R. Cohen and S. Havlin, Phys. Rev. Lett. 90, 058701 (2003).

[62] C. Buono and L. A. Braunstein, Europhys. Lett. 109, 26001 (2015).

[63] L. G. Alvarez Zuzek, C. Buono, and L. A. Braunstein, J. Phys.: Conf. Ser. 640, 012007 (2015). 